In der Rubrik „Literatur kompakt" werden die wichtigsten Originalarbeiten aus der internationalen Fachliteratur referiert.

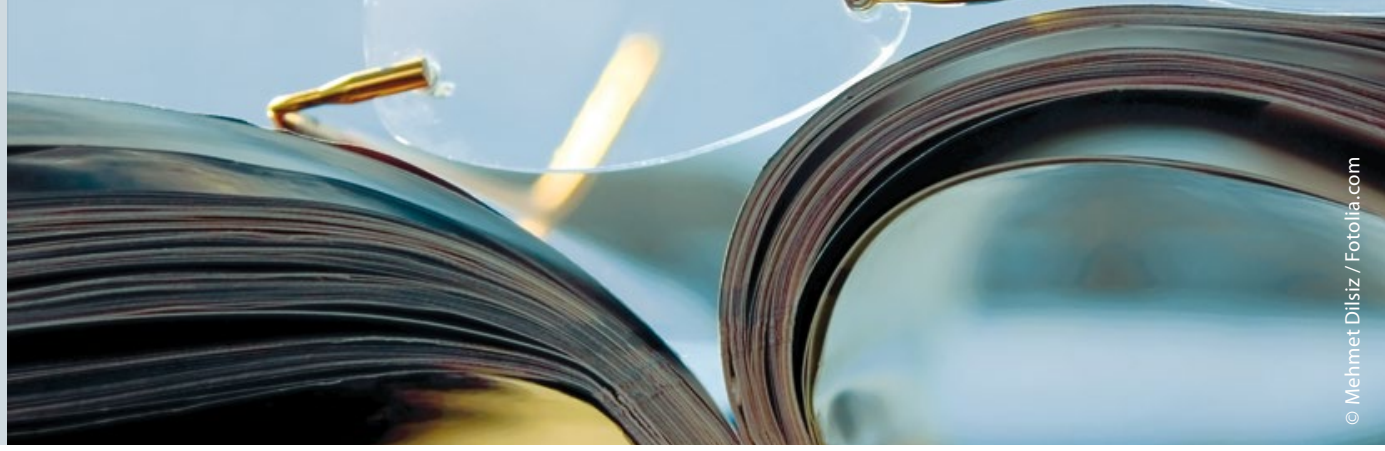

\title{
Opioid plus Benzo: Wie gefährlich ist die Kombi?
}

\section{Die Risiken einer Opioidtherapie in Kombination mit Benzodiazepinen wurden in einer großen US-Studie mit Kriegsveteranen untersucht.}

n den USA zählen Überdosierungen von Pharmazeutika zu den häufigsten Todesursachen. In zwei Dritteln dieser Fälle spielen Opioide eine Rolle. In Deutschland ist das Ausmaß dieses Problems weitaus geringer. Für die Schmerztherapie hierzulande ist jedoch ein Zusammenhang relevant, der sich jetzt zu bestätigen scheint: das erhöhte Mortalitätsrisiko unter einer Kombinationstherapie aus einem Opioid und einem Benzodiazepinpräparat.

Das Team um Tae Woo Park von der Brown University in Rhode Island hat, um diese Assoziation zu untersuchen, eine Fall-Kohortenstudie mit über 400.000 US-Kriegsveteranen durchgeführt. Von der Gesamtkohorte hatten 112.069 Teilnehmer in den Jahren 2004 bis 2009 sowohl ein Opioidanalgetikum als auch ein Benzodiazepinpräparat er- halten. Davon waren 2.400 an einer Überdosis des Analgetikums gestorben; 49\% dieser Todesfälle hatten sich in dem Zeitraum ereignet, in dem parallel ein Benzodiazepinrezept ausgestellt worden war.

Das Risiko einer tödlichen Opioidüberdosierung war bei gleichzeitiger Einnahme des Tranquilizers um das 3,86-Fache und bei früherer Einnahme um den Faktor 2,33 erhöht. Höhere Benzodiazepindosierungen waren mit einer höheren Anzahl von Todesfällen aufgrund einer Opioidüberdosierung verknüpft. Bei Betrachtung der einzelnen Substanzen galt die Risikoerhöhung für beinahe alle Arten von Benzodiazepinen, mit Ausnahme von Temazepam: Das Risiko einer tödlichen Überdosis Opioide war darunter um den Faktor 0,63 niedriger als bei Einnahme des am häufigsten verschriebenen Clonazepam.

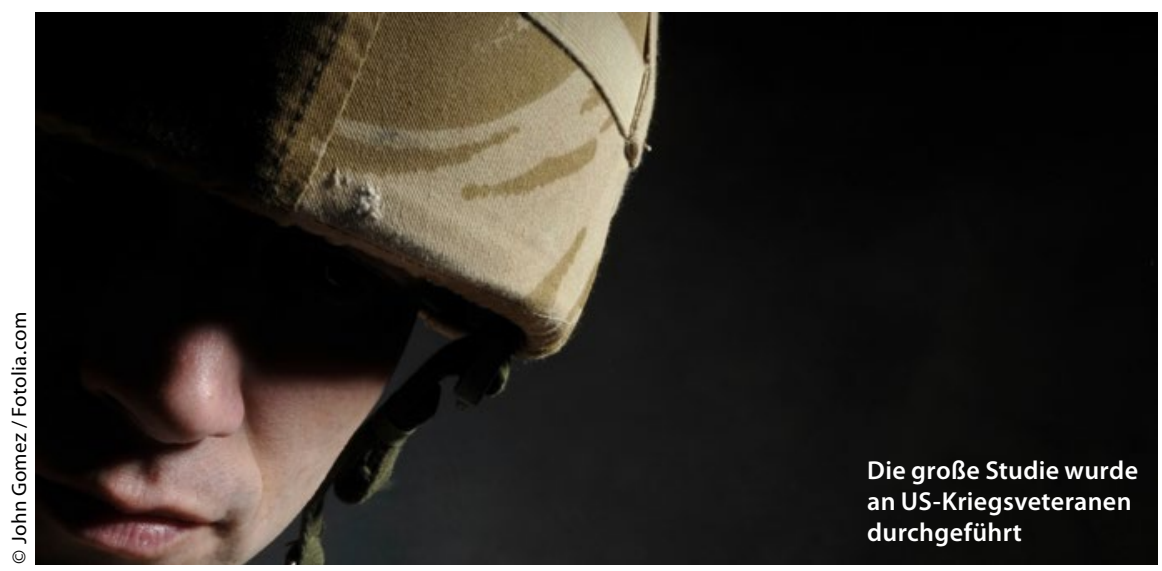

Benzodiazepine werden vielfach bei psychischen Störungen oder auch bei Substanzmissbrauch verschrieben. Tatsächlich litten von den Veteranen, die diese sedierenden, anxiolytisch und hypnotisch wirkenden Medikamente zusätzlich zu ihren Schmerzmedikamenten erhielten, $22 \%$ an einer posttraumatischen Belastungsstörung (PTSD), $20 \%$ an einer anderen Angststörung und $38 \%$ an einer Depression. Es liegt auf der Hand, dass Patienten mit einer solchen Erkrankung eher Probleme haben, mit dem Opioid verantwortungsvoll umzugehen. Eine Verzerrung der Ergebnisse wäre demnach zu erwarten. Die Forscher hatten jedoch, um dies zu vermeiden, eine Reihe von psychiatrische Diagnosen - vor allem PTSD, Angststörungen, Depressionen und bipolare Störungen - für die Endauswertung rechnerisch eliminiert, ebenso weitere potenzielle Einflussfaktoren wie sozioökonomischer Status und Diagnose einer Krebserkrankung. Der Zusammenhang blieb auch dann signifikant.

Fazit: Letztlich bleibt es unklar, inwieweit die Benzodiazepine zu den Todesfällen ursächlich beigetragen haben. Man sollte die Substanzen daher eher als „Risikomarker mit unbekanntem Kausalzusammenhang“ sehen, so der Vorschlag der Studienautoren. Angesichts der häufigen Komedikation mit Opioiden ist Vorsicht angebracht, insbesondere wenn höhere Dosen des Tranquilizers erforderlich sind. Elke Oberhofer

Park TW et al. Benzodiazepine prescribing patterns and deaths from drug overdose among US veterans receiving opioid analgesics: Case cohort study. BMJ 2015; 350: h2698; doi: 10.1136/ bmj.h2698 